\title{
Culturally-tailored Peer Support for Doctors and Nurses of Indian Origin
}

\begin{abstract}
The mental health and well-being needs of healthcare workers have risen sharply, fuelled by the current COVID-19 pandemic. Migrant healthcare workers are affected not only by events in the country where they work but also by events in countries where their family and friends reside. Despite, increase in psychological distress, migrant HCWs don't use the existing resources to support their needs.

This paper summarises discussions from the workshop focusing on reasons/barriers in uptake of mental health and well-being resources in the UK, the scope of peer support initiatives and its boundaries in supporting colleagues in the UK and India. Finally, it makes recommendations on the governance structure and checklist when organising peer support for healthcare workers.
\end{abstract}

Key words

Peer support; doctors in distress; BAPIO; health care workers

\section{Background}

The COVID-19 pandemic saw over 3.4 million lives lost and over 170 million people infected around the world, as of May 2021, according to figures from the World Health Organization [1]. Healthcare systems were under incredible strain with the overwhelming pressures. Healthcare workers (HCWs) across the world adapted rapidly in response to the crisis - even, at times, at cost to their physical and emotional wellbeing.

Immigrant doctors and nurses in the UK (making up between 22-37\% of the NHS workforce) were hit hard by the pandemic in their home countries despite planned easing of restrictions in the UK, with many loved ones affected by illness and mortality secondary to COVID-19. As a result, feelings of distress, helplessness and anger prevail amongst these colleagues [2]. Despite ongoing emotional turmoil and psychological distress, only a small proportion of these HCWs are utilising the health and wellbeing resources within the NHS [3]. The consequential impairment of wellbeing does impact on the ability of staff to cope with workload and may impair their ability to provide compassionate care [4]. Given that waiting times are amongst the longest recorded in recent history of the UK NHS, this is a serious cause for concern [5].

Mental health well-being of HCWs in India is compounded with lack of poor infrastructure and huge shortage of trained professionals leading to large treatment gap [6]. The pandemic has worsened the health and well-being status and various psychological resilience framework are proposed [7, 8]. As we prepare to tackle the aftermath of the pandemic, addressing staff fatigue and wellbeing should be a key

\begin{abstract}
Sunil Daga1, Viju
Varadarajan ${ }^{1}, \quad$ Manasvi Dwaraknath $^{2}$, Saraswati Hosdurga $^{1}$, Rajeev Metri ${ }^{3}$ and Rajeev Gupta ${ }^{4}$ on behalf of workshop delegates*
\end{abstract}

1.BAPIO Health and Well-being Forum, 2. BAPIO Young Doctors Forum, 3. British Indian Nursing Association (BINA); 4. National Forum for Health and Well-being (NFHW).

Contact

peersupport@bapio.co.uk

Cite as: Daga, S., Varadarajan, V., Dwaraknath, M., Hosdurga, S., Metri, R., Gupta, R. (2021) Culturally-tailored peer support for doctors and nurses of Indian origin. Sushruta J Health Policy vol 14; Issue 2:1-8 Art 10 https://doi.org/10.38192/14.2. $\underline{10}$

Article Information

Submitted 4.7.21

Revised 7.7.21

Published 8.7.21

ISSN 2732-5164 (Online)

ISSN 2732-5156 (Print)

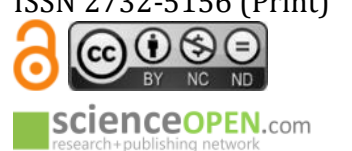


priority for all organisations. There is an unmet and urgent need to develop innovative, culturally appropriate and effective approaches to help overstretched and stressed HCWs.

\section{Problem statement}

Surveys of HCWs found that the prevalence of depression was $51 \%$, anxiety disorders $45 \%$, insomnia $34 \%$ and post-traumatic stress disorder (PTSD) 73\% post-pandemic [9]. A British Medical Association (BMA) analysis in April 2020 revealed that $30 \%$ of the 16,000 doctors surveyed reported a worsening in psychological and mental health since the start of the pandemic [10]. A further survey by the Nursing Times in May 2020 suggested that $33 \%$ of staff reported their overall mental health and wellbeing as "bad" or "very bad" and $50 \%$ described themselves as "a lot" more anxious or stressed than usual [11]. The Doctor's Association UK (DAUK) undertook a poll of 1,758 doctors across the UK - 65\% of respondents reported they would be leaving the NHS in the next three years and $45 \%$ cited the impact of the pandemic on their mental health as a driving factor in their considerations [12].

In August, NHS Providers surveyed 140 leaders from NHS Trusts and Foundations across England and almost all (99\%) said they were either moderately or extremely concerned by the current level of burnout across the workforce [13]. Researchers at King's College London surveyed staff in nine intensive care units across England about how they coped during the pandemic - $45 \%$ of the 709 respondents met the threshold for probable clinical significance for at least one of the following: PTSD (39\%), severe anxiety (11\%), problem drinking $(7 \%)$ and severe depression (6\%) [4]. There is no dearth of evidence demonstrating the negative impact that COVID-19 has had on the mental health of UK-based HCWs and the need for decision makers to take measures to address these both morally and from a productivity [14].

Similarly, a recent systemic review and metaanalysis from India reported $61 \%$ under stress, $43 \%$ anxious, $33 \%$ depression and $27 \%$ with sleep disturbances amongst Indian HCWs [7].

\section{Peer support}

Peer support is an inexpensive and effective approach in improving clinical care of chronic conditions [15]. As defined by the Mental Health Foundation, "Peer support is the help and support that people with lived experience of mental illness or a learning disability can give to one another' [16]. As defined by senior research associate for Advocates for Human Potential Inc., "a 'peer' is an equal, someone with whom one shares demographic or social similarities... 'Support' expresses the kind of deeply felt empathy, encouragement, and assistance that people with shared experiences can offer one another within a reciprocal relationship"[17].

Previous studies have demonstrated the importance of cultural competency in peer support programs, given that participants cite a sense of estrangement and insufficiently diverse composition (of peers) as reasons for not using the program [18]. The study also recognized the need of outreach and engagement strategies for the ongoing success of peer support programs. It is fundamental, therefore, that those providing peer support are trained and adequately supported.

The British Association of Physicians of Indian Origin (BAPIO) and its arm's length body, the British Indian Nursing Association (BINA) have a network extending their reach to many doctors and nurses based in the UK and in India. In collaboration with the National Forum for Health and Wellbeing (NFHW), the three groups have launched the i-Peer Support initiative aimed at defining, developing and delivering virtual peer support with following key objectives:

1. Exploring reasons for poor uptake of current health and wellbeing resources within the NHS

2. Defining culturally tailored peer support approaches

3. Defining boundaries of peer support both in the UK and India

4. Assessing the impact of the proposed peer support initiative

\section{Methodology}

Service development approaches were utilised with key stakeholders' participation. A multidisciplinary team (MDT) focus group with doctors and nurses from various specialties $(\mathrm{N}=15)$ explored causes of poor utilisation of the current health and wellbeing resources in the NHS. Subsequently, a workshop $(\mathrm{N}=29)$ with breakout discussions centred on the four aforementioned objectives was conducted, exploring the issues to great depth. Multidisciplinary participants (13 Male, 16 Female; 26 from South Asain background) were invited from all the three organisations (details of their role given in list of delegates). All participants contributed voluntarily and gave feedback to the final report. During the workshop, facilitator on each group made notes on what was 
discussed and then analysis was undertaken by two independent (SD and VV) members.

\section{Results}

\section{Reasons for poor uptake of current health and well-being resources in the NHS}

Participants expressed varied views on this; some felt this was a sensitive issue, both personally and culturally. References were made to upbringings where psychological health is not given the same priority as physical health and seeking help is considered taboo. Furthermore, these issues are compounded by mistrust and a poor perception of the existing infrastructure as well as an inability to relate to providers on a cultural level. Although cognizant of the significance of mental wellbeing, HCWs are not immune to the stigma associated with seeking help, fear of been judged and negative perception of colleagues. However, a safe and confidential space is considered imperative. The system may additionally lack a sensitive and understanding approach, with normalization of burnout and fear which is driving colleagues away from accessing resources.

\section{Quotes from participants regarding reasons for not accessing help:}

"Don't want to complain but be thankful and happy with what [they] have".

"If the language can be changed from 'gone off on stress leave' to 'need help or support', this allows a person to access help at earlier stage".

'In addition, there is a lack of awareness around accessing resources: whom to trust, when to talk and what to do." 'If we ask for help, we may be judged and thought to be unfit to do the job. We are also afraid of referral to the General Medical Council".

When I went to ask for help due to stress, I was told 'it's a stressful job; you shouldn't have chosen that speciality. I was made to feel this is entirely my mistake and it's my own problem" us."

"We do worry about what our colleagues or patients would think of

"A group of nurses who migrated to the UK felt lost and helpless".

HCWs from minority ethnic backgrounds are less comfortable seeking help for themselves out of a sense of stoicism. Awareness and navigation of the system is also poorly understood. Emotional and mental health awareness is still not the norm and managers may feel out of depth in addressing these issues.

\section{Defining culturally tailored peer support approaches}

Peer support should be a comfortable approach. Ability to connect with the listener who can understand the person's journey and values is important. Ability to relate culturally is the key, as better understanding and awareness of cultural norms is important. Poor perception of provider or support system by colleagues is an additional barrier. The current resources are felt either remote or too aligned to organisational objectives and not meeting their needs. 


\section{Quotes from participants around cultural aspects:}

"A person having gone through similar experiences and values can understand the feelings and be kind to the person seeking help. Moreover, the person seeking help get connected to a listener instantly".

"The opening statement of a peer, "I know what you are going through and I have been there and just come out of the other end", resonated immediately with me"

"I was asked to take a break and come out of the situation and send my ailing partner to respite. This is not accepted in my community and cultural practise".

\section{Defining boundaries of peer support}

Discussions on boundaries of peer support in India and UK had commonalities in terms of delivery aspects but there were specific challenges in context of India (detailed later).

3a. General consideration (applicable to UK and India)

Remit of Peer support and essential skill sets were discussed. The listening strategy facilitates those seeking support to reflect whilst encouraging and empowering them to come up with answers or solutions. When offering peer support to colleagues, the approach needs to be one of empathy and collectivism devoid of intimidation. This, however, could pose a challenge to those delivering the service unless trained to safely share their lived experiences, reinforcing once again the need to have proficiency and coaching for the providers.
Participants suggested highlighting at the start of any discussion what exactly we offer i.e., ease of access, confidentiality, an outside perspective or out-of-the-box thinking coupled with an ability and willingness to share personal lived experiences. There was a consensus that acknowledgement and validation of feelings is essential. Participants also highlighted the importance of placing emphasis on the fact that this is not a mental health service or a substitute for counselling.

Participants also discussed in detail the need for safeguarding mechanisms, particularly if someone is in acute distress and appears to be at risk to themselves or to others; it was suggested to signpost them appropriately, including writing to their health and wellbeing services at their employer or general practice or via private discussions with a peer who works in mental health services (e.g., psychiatrist, psychologist or a mental health nurse) with the individual's consent.

\section{Quotes from participants around boundaries of peer support:}

"A problem shared is a problem halved" and "emotional well-being of HCWs is everyone business."

"The approach taken by those delivering [peer support] should not be that of a 'know-it-all' individual but that of a modest, friendly colleague willing to share their own experience through listening and contributing sensibly". 
3b. Specific consideration for offering peer support to colleagues in India

In the context of settings in India specifically, there are no studies on peer support - this lack of data and evidence is a significant barrier. Participants expressed concerns about ability to support colleagues in India virtually and risks with not having clear and reliable signposting to resources or support network. Some suggested that it may be easier setting something on an institutional level rather than in the community initially. It was suggested that enlisting the help of psychiatrists from India and other support mechanisms in India beforehand is a priority of high importance.

\section{Quotes from participants around boundaries of peer support:}

"The lack of infrastructure and under-resourcing of healthcare systems poses a challenge to introducing structured peer support models in India".

"It's time for a change and if we see an active increase in demand for peer support due to the pandemic, this might act as a catalyst".

"How do we make sure their wellbeing is protected without access to necessary resources?"

\section{Assessing the impact of the proposed peer support initiative}

Impact assessment is paramount to appreciate the efficacy and many different potential methods were discussed. Subjective Unit of Distress Scale (SUDS) was suggested to assess intensity of feelings, with a rating of 0 corresponding to "bad" and a rating of 10 corresponding to "very good"- this was considered a simple option for administration and adherence [19]. This could be assessed individually before and relate the change following the sessions.

More detailed approaches such as a general health questionnaire (GHQ) that comprises 12 questions and the Harvard PAVING wheel to assess progress in wellbeing components were also suggested $[20,21]$. These could be useful tools in longitudinal self-assessment at regular intervals. However, such linked data at personal level cannot be obtained and analysed to assess the impact of peer support, as it is classified as sensitive information and goes against principle of confidentiality. This can only be done in research domain but can be used by individuals for self-evaluation over time.

\section{Discussion}

Support for HCWs has taken centre stage in the NHS Mental Health Implementation Plan 2019/20 and Health Education England is supporting this with the development of new roles and a competency framework for Peer Support Workers as a result. Peer support provision will serve as an important bridge between people facing psychological distress and mental health services and can reduce hospital admissions [22]. HCWs are experiencing high degrees of emotional distress for myriad reasons, which are further intensified due to the pressures of the COVID-19 pandemic. Additionally, immigrant HCWs are affected due to circumstances in their native countries, leaving them with feelings of anger, helplessness and guilt (when unable to provide direct help and support to their loved ones). Despite the need for and availability of health and wellbeing services at all NHS trusts, these are poorly accessed because of similar barriers to those faced by the general population [3]. There are also concerns that we are not adapting sufficiently quickly to meet mounting demands of the mental health and wellbeing crisis [23]. Peer support initiative for mental health is underutilised and many challenges exists in India [6].

A peer support network and associated programs can be cost-effective and are efficient resources secondary to their provider's relatability and trustworthiness. Most people in psychological distress require low levels of support, with few needing specialist services as outlined by the Stevenson/Farmer review of mental health and employers [24]. Several studies have demonstrated the success of peer support programs in 
responding to medical crises [25, 26]. More recent initiatives have been launched in the UK; nurses set up the Nurse Lifeline organisation with a view to offer 24/7 peer support to nurses and midwives. The British Medical Association (BMA) also launched a similar program for doctors and medical students [27].

We have conducted this workshop to establish a culturally sensitive peer support program for doctors and nurses of Indian origin through the collaborative efforts of BAPIO, BINA and NFHW, all three of which have a history of working with HCWs of South Asian communities. This paper ultimately describes the consensus position around various aspects of delivering such an initiative drawn from focus group and workshop discussions. We make the following recommendations:

1. The execution of this support network must be culturally tailored and based on peer support principles by bringing together colleagues with lived experience.

2. The initiative should provide a safe space with confidentiality (except in extreme circumstances to safeguard lives). The safe space will not ask for details of workplace, nor will any personal, sensitive or identifiable information be recorded or stored.

3. Provision should be voluntary, flexible with maximised accessibility in line with needs of colleagues

4. Provision can be virtually (via videoconferencing platforms) or face-to-face, taking into consideration pandemicrelated restrictions and comforts/preferences of users

5. When using existing NHS or third party mental health and wellbeing resources (such as mental health hub or board or private companies), it is important to have a collaborative approach with clear terms of reference/ memorandum of understanding (MOU) so as to incorporate the peer support groups within the safety of existing governance framework.

6. Good communication skills, particularly those of listening and reflective learning, would be essential for Peer support facilitators. If these are not part of their routine roles or they would like to refresh, they will be signposted to training courses by the BAPIO Training Academy.

7. The peer support initiative do not include provision of mental health services (no counselling or treatment offered), however:

a. The presence of a competent and trained mental healthcare professional in each session is required. This could be a psychologist or a medical/nursing colleague from the mental health services.

b. Identification of appropriate signposting and resources are required beforehand, for anyone who may require specific treatment or help.

c. Prior consent will be sought before formal referral for specialist intervention (counselling or treatment).

8. To complement peer support, a workbook will be developed to enable self-evaluation e.g., with the GHQ-12 and PAVING wheel exercises. The workbook will also list additional resources and signpost further help.

9. Impact of Peer Support sessions will be assessed by Subjective Unit of Distress Scale (SUDS).

\section{References}

1. Covid19.who.int. 2021 [cited June 2021]. WHO Coronavirus Dashboard. [online] Available at: <https://covid19.who.int/>.

2. Liberati E, Richards N, Willars J, et al. A qualitative study of experiences of NHS mental healthcare workers during the Covid-19 pandemic. BMC Psychiatry 2021;21.

3. Prajapati R, Liebling H. Accessing Mental Health Services: a Systematic Review and Meta-ethnography of the Experiences of South Asian Service Users in the UK. $J$ Racial Ethn Health Disparities 2021.

4. Greenberg N, Weston D, Hall C, et al. Mental health of staff working in intensive care during Covid-19. Occupational Medicine 2021;71(2):62-67. 
5. Gardner T, Fraser C. Longer waits, missing patients and catching up [Internet]. health.org.uk. 2021 [cited June 2021]. Available from: https://www.health.org.uk/news-andcomment/charts-and-infographics/howis-elective-care-coping-with-thecontinuing-impact-of-covid-19

6. Pathare S, Kalha J, Krishnamoorthy S. Peer support for mental illness in India: an underutilised resource. Epidemiol Psychiatr Sci 2018;27(5):415-419.

7. Singh R, Bajpai R, Kaswan P. COVID-19 pandemic and psychological wellbeing among health care workers and general population: A systematic-review and metaanalysis of the current evidence from India. Clin Epidemiol Glob Health 2021;11.

8. Banerjee D, Sathyanarayana Rao T, Kallivayalil R, et al. Psychosocial Framework of Resilience: Navigating Needs and Adversities During the Pandemic, A Qualitative Exploration in the Indian Frontline Physicians. Front Psychol 2021;12.

9. Lai J, Ma S, Wang Y, Cai Z, Hu J, Wei N et al. Factors Associated With Mental Health Outcomes Among Health Care Workers Exposed to Coronavirus Disease 2019. JAMA Netw Open 2020;3(3).

10. British Medical Association. BMA survey reveals almost half of doctors have relied upon donated or self-bought PPE and two thirds still don't feel fully protected. 2020 [cited June 2021]. Available from: https://www.bma.org.uk/bma-mediacentre/bma-survey-reveals-almost-half-ofdoctors-have-relied-upon-donated-or-selfbought-ppe-and-two-thirds-still-don-tfeel-fully-protected

11. Ford S. Exclusive: Nursing Times survey reveals negative impact of Covid-19 on nurse mental health. Nursing Times [Internet]. 2020 [cited June 2021]. Available from:

https://www.nursingtimes.net/news/men tal-health/exclusive-survey-revealsnegative-impact-of-covid-19-on-nursemental-health-29-04-2020/

12. Mass exodus of NHS doctors predicted by survey. ier.org.uk. 2020 [cited June 2021]. Available from: https://www.ier.org.uk/news/mass- exodus-of-nhs-doctors-predicted-bysurvey/

13. Ford M. Level of staff burnout 'real concern' for NHS trust leaders. Nursing Times [Internet]. 2020 [cited June 2021].. Available from: https://www.nursingtimes.net/news/wor kforce/level-of-staff-burnout-realconcern-for-nhs-trust-leaders-06-10$\underline{2020 /}$

14. Salari N, Khazaie H, Hosseinian-Far A, et al. The prevalence of stress, anxiety and depression within front-line healthcare workers caring for COVID-19 patients: a systematic review and meta-regression. Hum Resour Health 2020;18(1).

15. Wingate L, Graffy J, Holman D, et al. Can peer support be cost saving? An economic evaluation of RAPSID: a randomized controlled trial of peer support in diabetes compared to usual care alone in East of England communities. BMJ Open Diabetes Res Care 2017;5(1).

16. Peer support in mental health and learning disability [Internet]. London: Mental Health Foundation; 2012 [cited June 2021]. Available from: https://www.mentalhealth.org.uk/sites/d efault/files/need 2 know peer support1.p $\underline{\mathrm{df}}$

17. Penney D. Defining "Peer Support": Implications for Policy, Practice, and Research [Internet]. Sudbury: Advocates for Human Potential Inc.; 2018 [cited June 2021]. Available from:

https://www.ahpnet.com/AHPNet/media LAHPNetMediaLibrary/White\%20Papers/ DPenney_Defining_peer_support_2018_Fin al.pdf

18. Jonikas J, Kiosk S, Grey D, et al. Cultural competency in peer-run programs: Results of a web survey and implications for future practice. Psychiatr Rehabil J 2010;34(2):121-129.

19. Finch J. SUDS Thermometer. ccp.net.au. 2018 [cited June 2021]. Available from: https://ccp.net.au/suds-thermometer/

20. Goldberg D, Gater R, Sartorius N, et al. The validity of two versions of the GHQ in the WHO study of mental illness in general health care. Psychol Med 1997;27(1):191197. 
21. Frates B. PAVING the Path to Wellness. Boston: Harvard University; 2017 [cited June 2021]. Available from: https://www.rcsi.com/dublin//media/feature/media/downloaddocument/dublin/about/schoolsfaculties-and-departments/centre-forpositive-psychology-and-health/scienceof-health-and-happiness/rcsi-science-ofhappiness---paving-the-path-towellness.pdf

22. Repper J, Carter T. A review of the literature on peer support in mental health services. J Ment Health 2011;20(4):392411.

23. Docherty M. What has Covid-19 taught us about supporting workforce mental health and wellbeing? [Internet]. The King's Fund. 2020 [cited June 2021].. Available from: https://www.kingsfund.org.uk/blog/2020 /06/covid-19-supporting-workforcemental-health

24. Department for Work and Pensions, Department of Health and Social Care. Thriving at work: The Stevenson/Farmer review of mental health and employers. London: Public Health England; 2017 [cited June 2021]. Available from: https://www.gov.uk/government/publica tions/thriving-at-work-a-review-ofmental-health-and-employers

25. van Pelt F. Peer support: healthcare professionals supporting each other after adverse medical events. Qual Saf Health Care 2008;17(4):249-252.

26. Blake H, Gupta A, Javed M, et al. COVIDWell Study: Qualitative Evaluation of Supported Wellbeing Centres and Psychological First Aid for Healthcare Workers during the COVID-19 Pandemic. Int J Environ Res Public Health 2021;18(7).

27. Counselling and peer support for doctors and medical students [Internet]. bma.org.uk. 2021 [cited June 2021]. Available from:

https://www.bma.org.uk/advice-andsupport/your-wellbeing/wellbeingsupport-services/counselling-and-peersupport-services

\section{*Workshop delegates}

1. Dr Sunil Daga, iPeer Support Project Lead, BAPIO

2. Dr Viju Varadarajan, Member of National Executive committee, BAPIO

3. Dr Saraswati Hosdurga, Chair, BAPIO Health \& Well-being Forum

4. Dr Rajeev Gupta, Convener, NFHW and Chair, BAPIO Yorkshire

5. Dr Ramesh Mehta, President, BAPIO

6. Prof Romesh Gupta, Chairman, NFHW

7. Coumar Marimouttou, Chair, BINA

8. Dr Ananta Dave, President, BIPA

9. Dr Veena Daga, Director of Medical Education, BAPIO Training Academy

10. Prof Geeta Menon, Chair, BAPIO Faculty of Leadership

11. Dr Jyothi Srinivas, Chair, BAPIO Women's Forum

12. Mr Kantappa Gajanan, Chair, BAPIO SAS and LED forum

13. Prof Rob Campbell, Emeritus Professor of Philosophy at the University of Bolton

14. Bayo Igoh, Executive Coach \& Mentor

15. Jane Silver, Integrated-hypnopsychotherapist, Life and business coach

16. Dr Viji Saravanan, Consultant Psychiatrist

17. Reeny George Thomas, Senior Nurse

18. Dr Anand Jayaraman, Consultant Anaesthetist

19. Dr Kinnari Mehta, Consultant Anaesthetist

20. Ramesh Subbaih, Mental Health Nurse

21. Dr Umesh Prabhu, Ex-Medical Director in NHS

22. Dr Shital Godbole, General Practioner (GP)

23. Dr Sangeeta Nathdwarawala, Specialty Doctor in Psychiatry

24. Nisha Abraham, Speciality Nurse

25. Dr Gurunath Hosdurga, Consultant Anaesthetist

26. Dr Pawan Gupta, Psychiatrist

27. Dr Sriram Vaidyanathan, Consultant Radiologist

28. $\mathrm{Mr} \mathrm{C} \mathrm{R}$ Chandrasekar, Consultant Orthopaedics

29. Mr Yogesh Nathdwarawala, Consultant Orthopaedic Surgeon

Conflict of Interest: None declared 